\title{
Thermodynamics of Low-Dimensional Trapped Fermi Gases
}

\author{
Francisco J. Sevilla \\ Instituto de Física, Universidad Nacional Autónoma de México, Apdo. Postal 20-364, 01000 Ciudad de México, Mexico \\ Correspondence should be addressed to Francisco J. Sevilla; fjsevilla@fisica.unam.mx
}

Received 8 October 2016; Revised 2 December 2016; Accepted 5 December 2016; Published 26 January 2017

Academic Editor: Felix Sharipov

Copyright (C) 2017 Francisco J. Sevilla. This is an open access article distributed under the Creative Commons Attribution License, which permits unrestricted use, distribution, and reproduction in any medium, provided the original work is properly cited.

\begin{abstract}
The effects of low dimensionality on the thermodynamics of a Fermi gas trapped by isotropic power-law potentials are analyzed. Particular attention is given to different characteristic temperatures that emerge, at low dimensionality, in the thermodynamic functions of state and in the thermodynamic susceptibilities (isothermal compressibility and specific heat). An energy-entropy argument that physically favors the relevance of one of these characteristic temperatures, namely, the nonvanishing temperature at which the chemical potential reaches the Fermi energy value, is presented. Such an argument allows interpreting the nonmonotonic dependence of the chemical potential on temperature, as an indicator of the appearance of a thermodynamic regime, where the equilibrium states of a trapped Fermi gas are characterized by larger fluctuations in energy and particle density as is revealed in the corresponding thermodynamics susceptibilities.
\end{abstract}

\section{Introduction}

The discovery of the quantum statistics that incorporate Pauli's exclusion principle [1], made independently by Fermi [2] and Dirac [3], allowed the qualitative understanding of several physical phenomena-in a wide range of values of the particle density, from astrophysical scales to subnuclear ones-in terms of the ideal Fermi gas (IFG). The success of the explicative scope of the ideal Fermi gas model relies on Landau's Fermi liquid theory where fermions interacting repulsively through a short range forces can be described in some degree as an IFG. The situations change dramatically in low dimensions, since Fermi systems are inherently unstable towards any finite interaction [4-6]; thus the IFG in low dimensions becomes an interesting solvable model to study the thermodynamics of possible singular behavior.

On the other hand, the experimental realization of quantum degeneracy in trapped atomic Fermi gases [7-11] triggered a renewed interest, over the last fifteen years, in the study not only of interacting fermion systems [12-15] but also of trapped ideal ones [16-34]. Indeed, the nearly ideal situation has been experimentally realized by taking advantage of the suppression of $s$-wave scattering in spinpolarized fermion gases due to Pauli exclusion principle and of the negligible effects of $p$-wave scattering for the temperature ranges involved. Further, the control achieved on the experimental settings has opened the possibility of directly testing a variety of quantum effects such as Pauli blocking [35] and designing experiments to probe condensed matter models, though much lower temperatures are needed to achieve the phenomena of interest. On this trend, experimentally new techniques are being devised to cool further a cloud of atomic fermions [36-39]. Techniques based on the giving-away of entropy by changing the shape of the trapping potential have become of great importance and, as in many instances, a complete understanding of trapped noninteracting fermionic atoms would become of great value.

In distinction with the ideal Bose gas (IBG), which suffers the so-called Bose-Einstein condensation (BEC) in three dimensions, the IFG shows a smooth thermodynamic behavior as function of the particle density and temperature; this, however, does not preclude interesting behavior as has been pointed out in $[28,40]$, where it is suggested that the IFG can suffer a condensation-like process at a characteristic temperature $T^{0}$. Arguments based on a thermodynamic approach in support of this phenomenon are presented in [40], where the author suggests that the change of sign of the chemical potential, which defines the characteristic temperature $T^{0}$, marks the appearance of the condensed phase when the gas is cooled. 


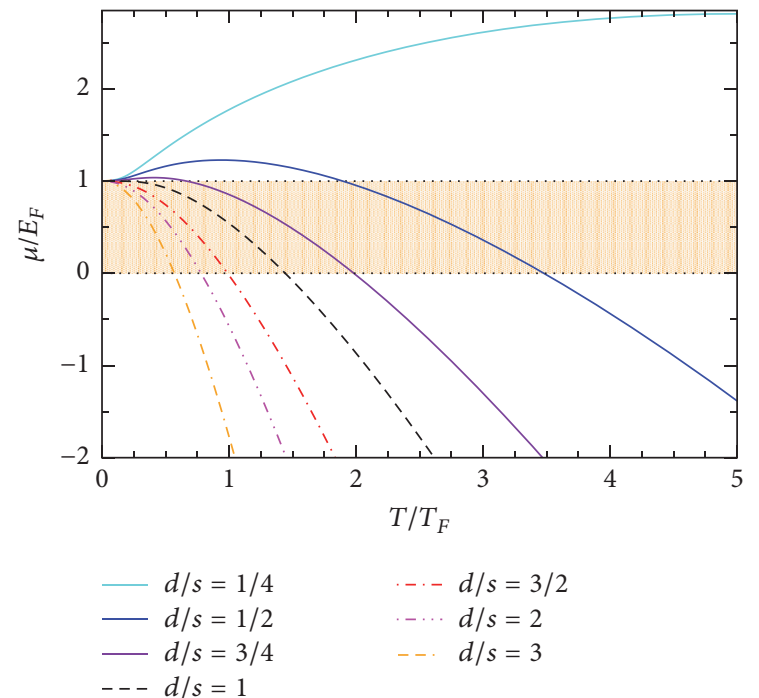

(a)

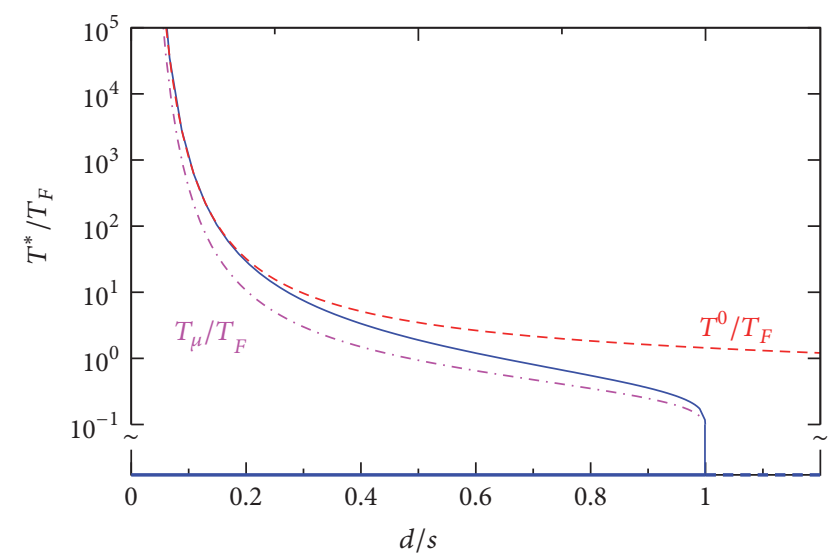

(b)

Figure 1: (a) Dimensionless chemical potential $\mu / E_{F}$ as function of the dimensionless temperature $T / T_{F}$ for different values of $d / s$. The crossings with the horizontal lines $\mu / E_{F}=1$ and $\mu / E_{F}=0$ mark the temperatures $T^{*}$ and $T^{0}$, respectively. (b) The temperature $T^{*}$ as function of $s / d$ (solid line). Additionally the temperatures $T^{0}$ (dashed line) and $T_{\mu}$ (dash-dotted line) are included for comparison.

Truly, the significance of $\mu$ has motivated the discussion of its meaning and/or importance at different levels and contexts [41-52]. For the widely discussed-textbook-case, namely, the three-dimensional IFG confined by an impenetrable box potential, the chemical potential results to be a monotonic decreasing function of the temperature, diminishing from the Fermi energy, $E_{F}$, at zero temperature, to the values of the ideal classical gas for temperatures much larger than $k_{B}^{-1}\left(\hbar^{2} / m \lambda_{T}^{2}\right)$, where $k_{B}$ is Boltzmann's constant, $\hbar$ is Planck's constant divided by $2 \pi, m$ is the mass of the particle, and $\lambda_{T}=\sqrt{2 \pi \hbar^{2} / m k_{B} T}$ is the thermal wavelength of de Broglie, where $T$ denotes the system's absolute temperature. A clear, qualitative, physical argument of this behavior is presented by Cook and Dickerson in [41]. In comparison, the chemical potential of the IBG vanishes below a characteristic temperature, called the critical temperature of BEC, $T_{c}$, and decreases monotonically for larger temperatures converging asymptotically to the values of the classical ideal gas.

This picture changes dramatically as the dimensionality of the system $d$ is lowered. In two dimensions the IBG shows no off-diagonal-long-range order at any finite temperature [53] and therefore the BEC transition does not occur. At this quirky dimension, the chemical potential of both, the Fermi and Bose ideal gases, decreases monotonically with temperature essentially in the same functional way [54], being different only by an additive constant, expressly, the Fermi energy. This results in the same temperature dependence of their respective specific heats at constant volume $C_{\mathscr{V}}$ [54-56]. In general, this last outstanding feature occurs whenever the number of energy levels per energy interval is uniform as in the case of a one-dimensional gas in a harmonic trap [57, 58], or the case $s=d$ where $s$ is the exponent of the single-particle energy spectrum of the form $\varepsilon \propto p^{s}, p$ being the particle momentum [59].

In one dimension, the chemical potential of the IBG decreases monotonically with temperature, and as in the two dimensional case, this behavior is related to the impossibility of BEC as shown by Hohenberg [53], at finite, nonzero, temperature. In contrast, the chemical potential of the IFG exhibits a nonmonotonic behavior: which starts rising quadratically with $T$ above the Fermi energy instead of decreasing from it and returns to its usual monotonic decreasing behavior at temperatures that can be as large as twice the Fermi temperature (see Figure 1; see also Figure 1 in [60]). This unexpected, and not well understood behavior, can be exhibited mathematically by the Sommerfeld expansion [60, 61] or by other methods [62-64], though no intuitive physical explanation of it, which predicts its appearance in the more general case, seems to have been given before. (Indeed, the precise argument presented in [41] is only valid for the free IFG in three dimensions.) This forms the basis for the motivation of the present paper.

After this excursus, one may conceive dimension two as a crossover value for which the thermodynamic properties of ideal quantum gases are conspicuously distinct for $d>2$ than those for $d<2$. This can be seen in the specific heat, which in the case of the IFG exhibits a no-bump $\rightarrow$ bump transition as dimension is varied from 3 to 1 [60] analogous to the wellknown cusp $\rightarrow$ no-cusp transition of the IBG specific heat. In the latter case, the cusp marks the BEC phase transition while no physical meaning is yet given for the bump in the former case.

In this paper we provide an analysis that attempts to explain the various features that are observed in the lowdimensional, trapped IFG, focusing in the nonmonotonic 
dependence on $T$ of the chemical potential. In Section 2 the system under consideration is described, thermodynamics quantities are calculated, and characteristics temperatures are introduced. In Section 3 a heuristic explanation of the nonmonotonic dependence of the IFG chemical potential on temperature is given. In Sections 4 and 5 the physical meaning of two relevant characteristic temperatures is given. Finally, conclusions and final remarks conform Section 6.

\section{General Relations, Calculation of the Chemical Potential, and the Thermodynamical Susceptibilities}

We consider an IFG of $N$, conserved, spinless fermions in arbitrary dimension $d>0$. We assume a single-particle density of states (DOS) of the form $[28,59]$

$$
g(\varepsilon)=G_{d, s} \varepsilon^{d / s-1},
$$

where $\varepsilon$ denotes the energy, $G_{d, s}$ and $s$ are positive constants, and the former depends on $d$ and on the specific energy spectrum of the system, while the latter is determined by the particular system dynamics.

Two instances lead to the power-law dependance in expression (1): the first one is based on the generalized energymomentum relation $[59,65] \varepsilon_{k}=\mathscr{C}_{s} k^{s}, k$ being the magnitude of the particle wave-vector $\mathbf{k}$ and $\mathscr{C}_{s}>0$ being a constant whose particular form depends on $s$. The physical cases $s=$ 2, 1 correspond, respectively, to the nonrelativistic IFG with $C_{2}=\hbar^{2} / 2 m$ and to the ultrarelativistic IFG for which $C_{1}=$ $c \hbar, c$ being the speed of light. In this case $G_{d, s}$ takes the form $\mathscr{V} /\left[2^{d-1} \pi^{d / 2} \Gamma(d / 2) s C_{s}^{d / s}\right]$, with $\Gamma(\sigma)$ the gamma function and $\mathscr{V}=L^{d}$ the volume of the system. The second instance is based on the $d$-dimensional IFG trapped by an isotropic potential of the form $U(\mathbf{r})=U_{0}\left(r / r_{0}\right)^{\alpha}$, where $U_{0}, r_{0}$ are two constants that characterize the energy and length scales of the trap. This trapping potential leads, in the semiclassical approximation [66], to $G_{d, s}=((2 / s-1) \Gamma[d(1 / s-$ $\left.1 / 2)] / \Gamma(d / 2) \Gamma[d / s] \hbar^{d}\right)\left(m r_{0}^{2} / 2\right)^{d / 2} U_{0}^{d(1 / 2-1 / s)}$ with $s^{-1}=1 / 2+$ $\alpha^{-1}$. Notice that in the latter case, one can immediately establish the thermodynamic equivalence between the IBG and the IFG; namely, $\alpha=2 d /(2-d)$, implying that no such equivalence is possible in dimensions $d>2$ for positive $\alpha$. The equivalence does occur in two dimensions if $\alpha \rightarrow \infty$, which corresponds to the infinite well potential and in one dimension if $\alpha=2$, which corresponds to the harmonic potential.

The thermodynamical properties of the ideal quantum gases are easily computed from the grand potential $\Omega(T, \mathscr{V}, \mu) \equiv U-T S-\mu N[67,68]$, where $U$ and $S$ denote the internal energy and entropy, respectively. For the trapped gas, $\mathscr{V}$ denotes the appropriate thermodynamic variable that generalizes the volume of a fluid in a rigid-walls container (see [69] for the case of the three-dimensional harmonic trap), which in this paper is taken as $\mathscr{V}=\left(m r_{0}^{2} / 2\right)^{d / 2} U_{0}^{d(1 / 2-1 / s)}$ which reduces to $\mathscr{V}=\omega^{-d}$ for the isotropic harmonic trap $U(\mathbf{r})=\hbar \omega\left(r / r_{0}\right)^{2}$ with $r_{0}=(2 \hbar / m \omega)^{1 / 2}$. For a gas of noninteracting fermions, $\Omega(T, \mathscr{V}, \mu)$ can be written in the thermodynamic limit, $N \rightarrow \infty$ and $\mathscr{V} \rightarrow \infty$ with $N / \mathscr{V}=$ constant, [70] as

$$
\begin{aligned}
\Omega(T, \mathscr{V}, \mu)= & -k_{B} T \int_{0}^{\infty} d \varepsilon g(\varepsilon) \\
& \times \ln \left[\exp \{\beta(\varepsilon-\mu)\} f_{\mathrm{FD}}(\varepsilon, T)\right],
\end{aligned}
$$

where $f_{\mathrm{FD}}(\varepsilon, T)=\{\exp [\beta(\varepsilon-\mu)]+1\}^{-1}$ is the Fermi-Dirac distribution function that gives the average occupation of the single-particle energy state $\varepsilon$ at absolute temperature $T$. As usual, $\beta$ denotes the inverse of the product of $T$ and $k_{B}$ Boltzmann's constant.

The average number of fermions $N(T, \mathscr{V}, \mu)$ in the system is given by $-(\partial \Omega / \partial \mu)_{T, \mathscr{V}}$ [67] which gives

$$
\frac{N}{\mathscr{V}}=-\bar{G}_{d, s} \Gamma\left(\frac{d}{s}\right)\left(k_{B} T\right)^{d / s} \operatorname{Li}_{d / s}\left(-e^{\beta \mu}\right),
$$

where $\operatorname{Li}_{\sigma}(z)=\sum_{l=1}^{\infty} z^{l} / l^{\sigma}$ is the polylogarithm function of order $\sigma$ [71] and $\bar{G}_{d, s}=G_{d, s} / \mathscr{V}$. Expression (3) relates $N$ and $\mu$, and for fixed $N$, the chemical potential is a function of the system temperature and volume. The internal energy $U(T, \mathscr{V}, \mu)$ per volume is given by

$$
\frac{U}{\mathscr{V}}=-\bar{G}_{d, s} \Gamma\left(\frac{d}{s}+1\right)\left(k_{B} T\right)^{d / s+1} \operatorname{Li}_{d / s+1}\left(-e^{\beta \mu}\right),
$$

while the entropy $S(T, \mathscr{V}, \mu)=-(\partial \Omega / \partial T)_{\mathscr{V}, \mu}$ per volume by

$$
\begin{aligned}
\frac{S}{\mathscr{V}} & =-k_{B} \bar{G}_{d, s} \Gamma\left(\frac{d}{s}\right)\left(k_{B} T\right)^{d / s} \\
\cdot & {\left[\left(\frac{d}{s}+1\right) \times \operatorname{Li}_{d / s+1}\left(-e^{\beta \mu}\right)-\frac{\mu}{k_{B} T} \operatorname{Li}_{d / s}\left(-e^{\beta \mu}\right)\right] . }
\end{aligned}
$$

In Figure 1(a) the temperature dependence of the ratio $\mu / E_{F}$ is shown for different values of the ratio $d / s$ and for $N / \mathscr{V}$ fixed, where $T_{F}$ denotes the Fermi temperature defined through the relation $E_{F}=k_{B} T_{F}$, where $E_{F}$ is explicitly given by $\left(d / s \bar{G}_{d, s}\right)^{s / d}(N / \mathscr{V})^{s / d}$ in $d$ dimensions. For $d / s<1$ the nonmonotonic dependence on temperature is clearly shown (the dashed line corresponds to the case $d / s=1 / 2$, while $d / s=1 / 4$ is presented with the only purpose of making the effects of the system dimensionality more conspicuous). In the limit of high temperatures, $T \gg T_{F}$, the classical result $\mu \rightarrow k_{B} T \ln \left[\left(T / T_{F}\right)^{d / s} \Gamma(d / s+1)\right]$ is recovered.

As occurs for the $2 \mathrm{D}$ ideal gas in a box potential $(s=$ $d=2$ ), the DOS is a constant whenever $s=d$, and the chemical potential has the well-known analytical dependence on the temperature $\mu=E_{F}+k_{B} T \ln \left[1-e^{-T_{F} / T}\right]$. For $T \ll$ $T_{F}$, the chemical potential lies below the Fermi energy by a negligible, exponentially small correction. The low temperature behavior of $\mu$ for $d \neq s$ can be obtained approximately as a direct application of the Sommerfeld expansion for $T \ll T_{F}$ (see [72, pp. 45-46]); namely,

$$
\mu \simeq E_{F}\left[1-\frac{\pi^{2}}{6}\left(\frac{d}{s}-1\right)\left(\frac{T}{T_{F}}\right)^{2}\right]+\mathcal{O}\left(\left[\frac{T}{T_{F}}\right]^{4}\right) .
$$


The power-law dependence on $\varepsilon$ in expression (1) is manifested itself in the last expression, where the ratio $d / s$ appears explicitly. Clearly, for $d / s<1$, the chemical potential rises from the Fermi energy quadratically with $T$, and the nonmonotonousness is a result of the fact that, for large enough temperatures, $\mu(T)$ falls down with temperature to negative values close to those of the classical gas. As a consequence of this "turning around," $\mu(T)$ develops a maximum at temperature $T_{\mu}$ and equals $E_{F}$ at two distinct temperatures, at $T^{*}$ and 0 , if $d / s<1$, and only at $T=0$ otherwise. Thus, the solution to the equation $\mu(T)=E_{F}$ as function of the parameter $d / s$ bifurcates at the critical value $d=s$ as is shown in Figure 1(b). Note that, for $s=2$ and $d=1, T^{*}$ is as large as $1.896 T_{F}$ and diverges as $d / s \rightarrow 0$. This can be shown straightforwardly from (3) by putting $\mu=E_{F}$, since then $T^{*}$ must satisfy the equation $1=\left[1+e^{-T_{F} / T^{*}}\right]^{-1}$ in that limit.

In addition, the temperatures $T^{0}$ and $T_{\mu}$ that mark the change of sign of $\mu$ and its maximum, respectively, are also shown in Figure 1(b) (dashed line and dashed-dotted line). $T^{0}$ is determined from the equation $\mu\left(T^{0}\right)=0$, which explicitly gives

$$
T^{0}=\left[\Gamma\left(\frac{d}{s}+1\right) \zeta\left(\frac{d}{s}\right)\left(1-2^{1-d / s}\right)\right]^{-s / d} T_{F}
$$

this expression gives the approximated values $3.48 T_{F}, 1.44 T_{F}$, and $0.989 T_{F}$ for $d / s=1 / 2,1,3 / 2$, respectively. The temperature $T^{0}$ diverges as $\exp \{(s / d) \ln 2\}$ as $d / s \rightarrow 0$ and goes to zero as $[e /(d / s)] / \sqrt{2 \pi d / s}^{s / d}$ as $d / s \gg 1$, where $e$ is the EulerNapier number.

It is clear from expression (6) that $d<s$ is required for the anomalous behavior of $\mu(T)$ to take place; however, for physical systems with positive integer dimensions less than three impose severe restrictions on how fast the trapping potential must grow with the system size, that is, on the values of the exponent $\alpha$. For fermions in a box-like trap $(s=2)$ the anomaly will be observed if $d=1$, a case where the effects are conspicuously revealed even at large temperatures. This case indeed poses a challenge to trap designing, though, it could be realized experimentally by using the optical trap developed by Meyrath et al. [73]. In the typical experimental situation of harmonically trapped Fermi gases $(\alpha=2$ and therefore $s=1$ ) studied intensively $[16,23,26,31]$ expression (6) tells us that the anomaly is not observed for any integer $d \geq 1$. On the other hand, if one assumes $d=1$ as the minimum system dimensionality realizable experimentally (cigar shaped traps), then one should go beyond harmonic trapping; that is, one has to choose $\alpha>2$.

The nonmonotonicity of the chemical potential, just referred to during the previous paragraphs, is revealed in the thermodynamic susceptibilities. In this work we focus on the specific heat at constant volume $C_{\mathscr{V}}=(\partial U / \partial T)_{\mathscr{V}}$ and the isothermal compressibility $\kappa_{T}=\left(1 / n^{2}\right)(\partial n / \partial \mu)_{T}$, given by

$$
\begin{aligned}
\frac{C_{\mathscr{V}}}{N k_{B}}= & \frac{d}{s}\left(\frac{d}{s}+1\right) \frac{\mathrm{Li}_{d / s+1}\left(-e^{\beta \mu}\right)}{\operatorname{Li}_{d / s}\left(-e^{\beta \mu}\right)} \\
& -\left(\frac{d}{s}\right)^{2} \frac{\mathrm{Li}_{d / s}\left(-e^{\beta \mu}\right)}{\operatorname{Li}_{d / s-1}\left(-e^{\beta \mu}\right)},
\end{aligned}
$$

TABLE 1: The temperatures $T^{0}, T^{*}, T_{\mu}, T_{C_{\mathscr{V}}}$, and $T_{\kappa_{T}}$ for which $\mu\left(T^{0}\right)=0, \mu\left(T^{*}\right)=E_{F}, \mu\left(T_{\mu}\right)$ is maximum, $C_{\mathscr{V}}\left(T_{C_{\mathscr{V}}}\right)$ is maximum, and $\kappa_{T}\left(T_{\kappa_{T}}\right)$ is maximum, for three characteristic values of $d / s$, namely, $1 / 4,1 / 2$, and $3 / 4$, at which a nonmonotonic behavior is observed.

\begin{tabular}{lccccc}
\hline$d / s$ & $T^{0}$ & $T^{*}$ & $T_{\mu}$ & $T_{C_{\mathscr{V}}}$ & $T_{\kappa_{T}}$ \\
\hline 0.25 & 15.6729 & 13.2260 & 5.0286 & 0.6532 & 0.3751 \\
0.5 & 3.4797 & 1.8960 & 0.9365 & 0.8632 & 0.2906 \\
0.75 & 1.9830 & 0.6666 & 0.4086 & 1.3893 & 0.2080 \\
\hline
\end{tabular}

$$
\kappa_{T}=\frac{\mathscr{V}}{N k_{B} T} \frac{\operatorname{Li}_{d / s-1}\left(-e^{\beta \mu}\right)}{\operatorname{Li}_{d / s}\left(-e^{\beta \mu}\right)},
$$

respectively.

In Figure 2 the dimensionless $C_{\mathscr{V}}(T) s / d N k_{B}$ (a) and $\kappa_{T} / \kappa_{0}$ (b) are shown as function of the dimensionless temperature $T / T_{F}$ for different values of $d / s$; clearly, for $d / s<$ 1 , both quantities exhibit a nonmonotonous dependence on $T$. The specific heat clearly exhibits the universal linear dependence on $T$ in the low temperature regime and rises with temperature evidencing the effects of dimensionality. In the high temperature regime all the curves converge to the classical result $d N k_{B} / s$. Analogously, the isothermal compressibility exhibits the universal behavior in the low temperature regime, namely, a finite value due to the degeneracy pressure. As temperature rises the effects of dimensionality are uncovered but are hidden again in the high temperature regime, where the classical dependence on temperature appears.

The nonmonotonic dependence with temperature of both thermodynamic susceptibilities is manifested as a global maximum at the temperatures $T_{C_{\mathscr{V}}}$ and $T_{\kappa_{T}}$, respectively (see solid lines in Figures 2(a) and 2(b)). One would be tempted to propose that either of these temperatures would distinguish between two distinct behaviors of the IFG: one where the corresponding susceptibility behaves anomalously and the other where it behaves standardly. Notice, nevertheless, that such temperatures do not match between them or with either the temperature $T_{\mu}$ or the temperature $T^{*}$, as can be quantitatively appreciated in Table 1 and in Figure 2, where solid triangles in both panels identify the values of the corresponding susceptibility evaluated at $T_{\mu}$, solid circles in (a) indicate the values of $C_{\mathscr{V}}$ at $T_{\kappa_{T}}$, and, analogously, solid squares in (b) mark the value of $\kappa_{T}$ at $T_{C_{\mathscr{V}}}$ for $d / s=$ $1 / 4,1 / 2$, and $3 / 4$. Such discrepancy among all these temperatures makes it difficult to consider them as points that mark the separation of two distinct thermodynamic behaviors.

For the signal value $d / s=1$, expressions in terms of elementary functions are possible (black-dashed lines in Figure 2); namely,

$$
\frac{C_{\mathscr{V}}}{N k_{B}}=2 \frac{\operatorname{Li}_{2}\left(e^{\beta \mu}\right)}{\ln \left(1+e^{\beta \mu}\right)}-\left(1+e^{-\beta \mu}\right) \ln \left(1+e^{\beta \mu}\right)
$$




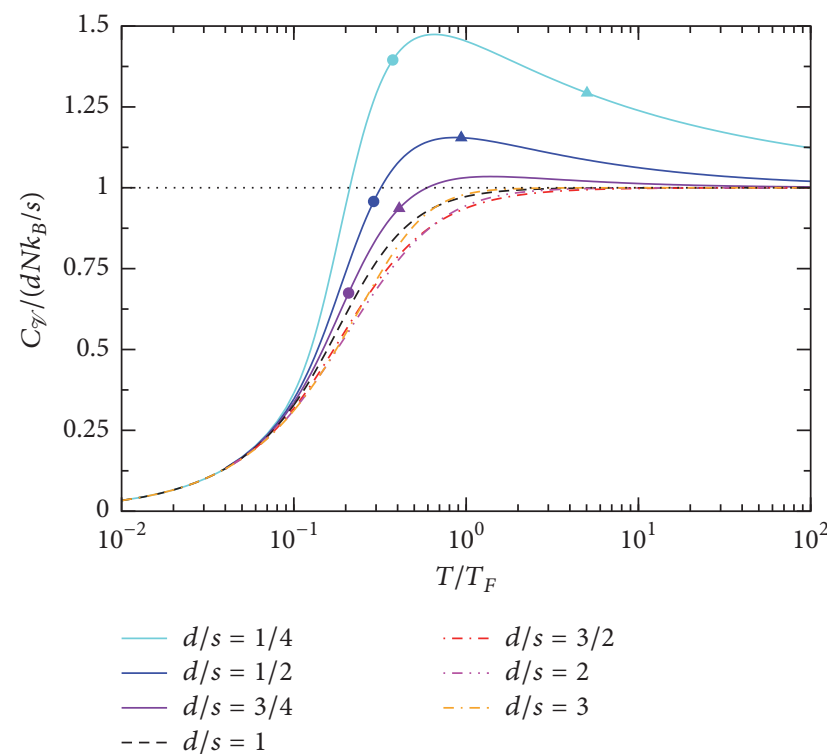

(a)

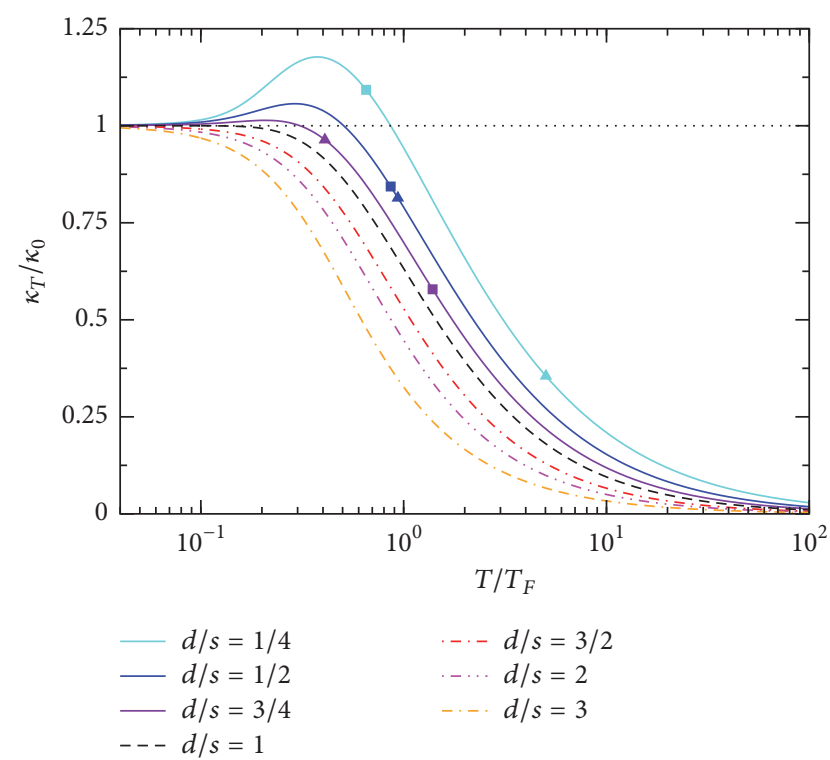

(b)

Figure 2: Normalized thermodynamic susceptibilities as function of the dimensionless temperature $T / T_{F}$ for different values of $d / s$, say, $1 / 4,1 / 2,3 / 4,1,3 / 2,2$, and 3. (a) Specific heat per particle at constant generalized volume; the circles mark the corresponding values of $C_{\mathscr{V}}$ at $T_{\kappa_{T}}$, that is, at the temperature at which $\kappa_{T}$ has a maximum value; analogously, the triangles do the same at $T_{\mu}$ where $\mu$ has a maximum. (b) Isothermal compressibility scaled with $\kappa_{0}=(d / s)^{2}\left(\pi^{d} \Gamma(d / 2) / 2 \pi^{d / 2}\right) s C_{s}^{d / s} E_{F}^{-(d / s+1)} ;$ rhombus marks the corresponding values of $\kappa_{T}$ at $T_{C_{\mathscr{V}}}$ that corresponds to the temperature at which $\kappa_{T}$ has a maximum value and the triangles do the same as in (a). Notice the nonmonotonic dependence on $T$ for $d / s<1$.

$$
\kappa_{T}=\frac{\mathscr{V}}{N k_{B} T} \frac{e^{\beta \mu}}{\left(1+e^{\beta \mu}\right) \ln \left(1+e^{\beta \mu}\right)},
$$

where we have used the fact that the polylogarithm functions of order $0,1,2$ correspond to the elementary functions $\mathrm{Li}_{0}(z)=\ln (1+x), \operatorname{Li}_{1}(z)=z / 1+z$, and $\operatorname{Li}_{2}(z)=$ $\int_{0}^{z} x \mathrm{~d} x / 1+x$, respectively. For $d / s>1$, the variation with temperature of the thermodynamic susceptibilities is standard.

\section{Heuristic Explanation of the Nonmonotonic Dependence of $\mu$ on $T$ for $d / s<1$}

The monotonic decreasing behavior of the chemical potential with temperature for $d / s \geq 1$ is understood from the argument based on the fact that the internal energy $U$ diminishes from its zero temperature value $E_{F}$ after adiabatically adding a fermion at the small temperatures $T \ll T_{F}$. Quoting Cook and Dickerson [41], the system cools by redistributing the particles into the available energy states in such a way that the particle added goes into "... a low lying, vacant single particle state, which will be a little below $E_{F}$," This is a consequence, as we will show below that in the three-dimensional case the change of the Helmholtz free energy is dominated by the change of entropy in the low temperature limit; however, the argument provided in [41] does not give the amount of the energy change involved in the process or the change in temperature, making the nature of the argument just qualitative. In fact, the difficulty in quantifying those quantities arises from the use of the thermodynamic relation

$$
\mu(S, \mathscr{V}, N)=\left(\frac{\partial U}{\partial N}\right)_{S, \mathscr{V}}
$$

which requires the knowledge of $U(S, \mathscr{V}, N)$, rarely considered for analysis in the variables $S, \mathscr{V}, N$. From (2) the functions $N=N(\mu, T, \mathscr{V})$ (3) and $S=S(\mu, T, \mathscr{V})$ (5) are obtained and solved in order to obtain $U(S, \mathscr{V}, N)$. In Figure 3 the internal energy at constant entropy is plotted as function of the particle density $N / \mathscr{V}$ for $S / k_{B} \mathscr{V}=0.1$ and for different values of the ratio $d / s$. The slope of the curves gives the value of the chemical potential as given by expression (10). Also in the same Figure 3, but in (b), the temperature of the system, scaled with the Fermi temperature, as function of the particle density is shown for $S / k_{B} \mathscr{V}=0.1$. Clearly, the systems cool regardless of the ratio $d / s$, when adding particles to the system in an isentropic way.

It is possible to obtain an expression for $\mu(S, \mathscr{V}, N)$ from (10) by the use of the asymptotic behavior of the Polylogarithm functions $-\operatorname{Li}_{\sigma}(-z) \simeq \ln (z)^{\sigma} / \Gamma(\sigma+1)+\left(\pi^{2} /\right.$ 6) $\ln (z)^{\sigma-2} / \Gamma(\sigma-1)+\cdots$; after some algebra we have expressed that in the degenerate regime

$$
\begin{aligned}
& \mu(S, \mathscr{V}, N) \\
& \quad \simeq E_{F}\left[1+\frac{3}{2 \pi^{2}}\left(\frac{S}{k_{B} \mathscr{V}}\right)^{2}\left(\frac{\mathscr{V}}{N}\right)^{2} \frac{s}{d}\left(\frac{s}{d}-1\right)\right],
\end{aligned}
$$




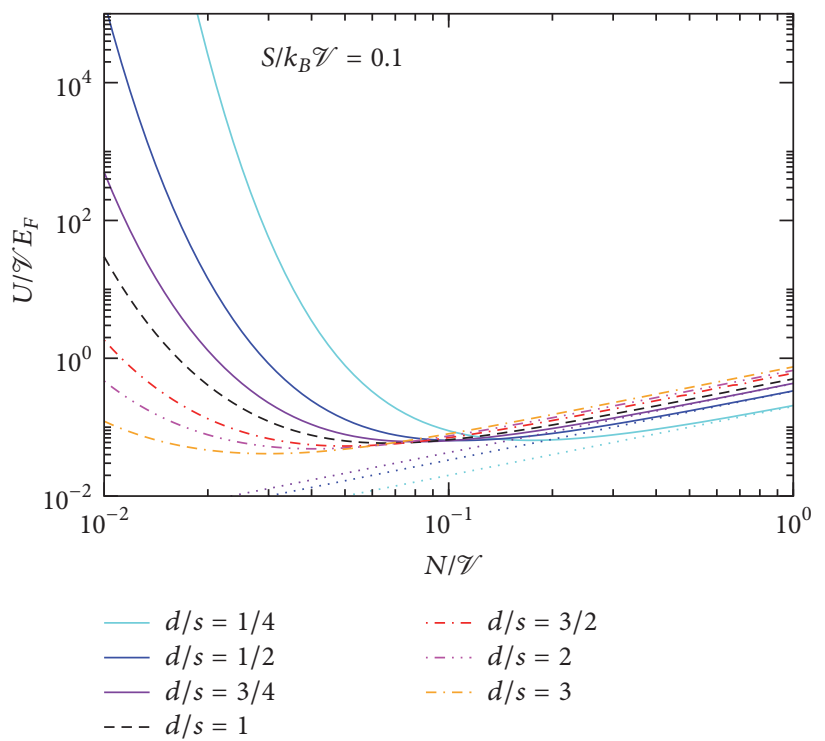

(a)

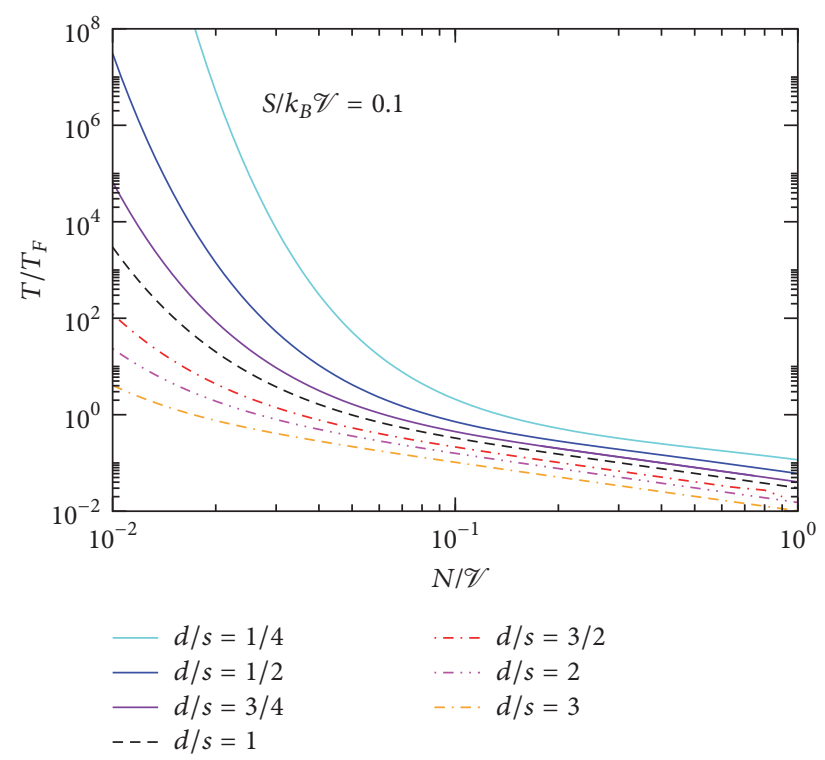

(b)

FIGURE 3: The dependence of the scaled internal energy $U / \mathscr{V} E_{F}(a)$ and scaled temperature $T / T_{F}(b)$ as function of the dimensionless particle density $N / \mathscr{V}$, where $\mathscr{V}$ denotes the systems volume scaled with an arbitrary volume $\mathscr{V}_{0}$.

where the nonmonotonic dependence on $T$ is evident when $d / s<1$. On the other hand, for the sake of completeness we compute the system temperature as function of the particle density, in the degenerate limit, which is given by

$$
T(S, \mathscr{V}, N) \simeq \frac{3}{\pi^{2}} \frac{s}{d} T_{F} \frac{S}{k_{B} \mathscr{V}} \frac{\mathscr{V}}{N}
$$

and, as is shown in Figure 3(b), decreases as $(N / \mathscr{V})^{-1}$.

How can we understand the rising of the chemical potential when $d / s<1$ ? Consider the number of particles that can be excited by the energy $k_{B} T \ll E_{F}$ from the $d$-dimensional Fermi sphere. This number is approximately given by $N k_{B} T / E_{F}$ while the number of available states above the Fermi energy can be approximated by $g\left(E_{F}\right) k_{B} T$. The quotient between both quantities is exactly $s / d$. This simple and heuristic argument shows that there are more singleparticle excited states than excitable particles for $d / s>1$, which is evident because of the monotonic increasing behavior of the DOS. In principle all the excited particles can be accommodated into the available states without violating Pauli's principle. The accommodation, however, is not arbitrary. The probability of occupation of the available states in thermal equilibrium must follow the Fermi-Dirac distribution and therefore just a fraction of the excitable fermions are excited into the interval $\left[E_{F}, E_{F}+k_{B} T\right]$ (in fact, the occupation probability for the states with energy larger than $\mu$ is smaller than 1/2). For this case we can certainly apply the argument given by Cook and Dickerson in [41] to infer that when adding adiabatically an extra particle to the system, the internal energy will decrease from $E_{F}$.

In contrast, Pauli exclusion principle prohibits complete accommodation when $d / s<1$, since in this case the DOS has a monotonic decreasing dependence on energy and, as a consequence, the number of available excited states is reduced considerably in comparison with excitable number of particles. We may conclude that when adding a particle in an adiabatically way, the probability of occupying an energy state below $E_{F}$ is very small and therefore, it will occupy an energy state above $E_{F}$.

In order to quantitatively characterize the incomplete accommodation described above, we consider the ratio $R(T)$ of the number of particles in the energy interval $\left[E_{F}, E_{F}+\Delta\right]$ to the number of available states in the same energy interval,

$$
R(T)=\frac{\int_{E_{F}}^{E_{F}+\Delta} d \varepsilon g(\varepsilon) f_{\mathrm{FD}}(\varepsilon, T)}{\int_{E_{F}}^{E_{F}+\Delta} d \varepsilon g(\varepsilon)} .
$$

This quantity is shown in Figure 4 as function of temperature with ratio $\Delta / E_{F}=0.001$, for different values of $d / s$. The choice $\Delta \sim k_{B} T \ll E_{F}$ guarantees that a negligible number of particles occupy states out of the interval $\left[E_{F}, E_{F}+\Delta\right]$. Under this condition we can approximate $R(T)$ by $f_{\mathrm{FD}}\left(E_{F}, T\right)$ and for temperatures $0<T \ll T_{F}$ we have that $R(T) \simeq(1 / 2)[1-$ $\left.\left(\pi^{2} / 12\right)(d / s-1)\left(T / T_{F}\right)\right]$, and, therefore, the occupation probability of the energy states in $\left[E_{F}, E_{F}+\Delta\right]$ is smaller than $1 / 2$ for $d / s>1$, greater than $1 / 2$ for $d / s<1$, and equal to $1 / 2$ for $d=s$.

\section{The Physical Meaning of $T^{0}$}

A condensation-like phenomenon has been suggested to occur in the IFG in $[28,40]$; this can be understood as the formation of a "core" in momentum-space, reminiscent of the Fermi sea, that starts forming at $T^{0}$ and that grows up to form the Fermi sea as temperature is diminished to absolute zero. The number of particles in the core, $n_{\text {core }}$, is computed 


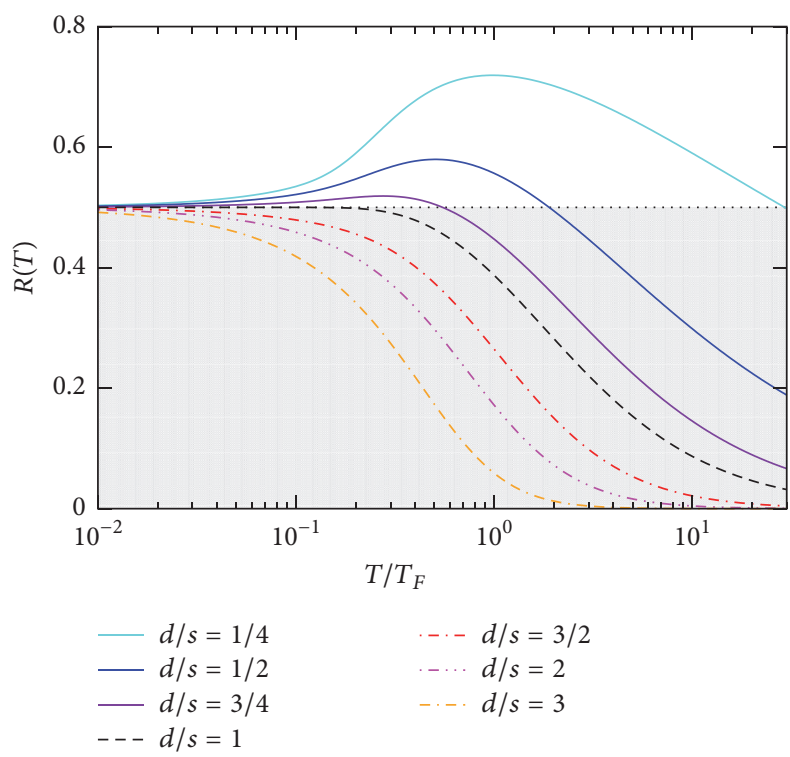

FIGURE 4: The ratio of the number of particles in the energy interval $\left[E_{F}, E_{F}+\Delta\right]$ to the number of available states in the same energy interval, $R(T)$ (see (13)), as function of temperature for different values of $d / s$.

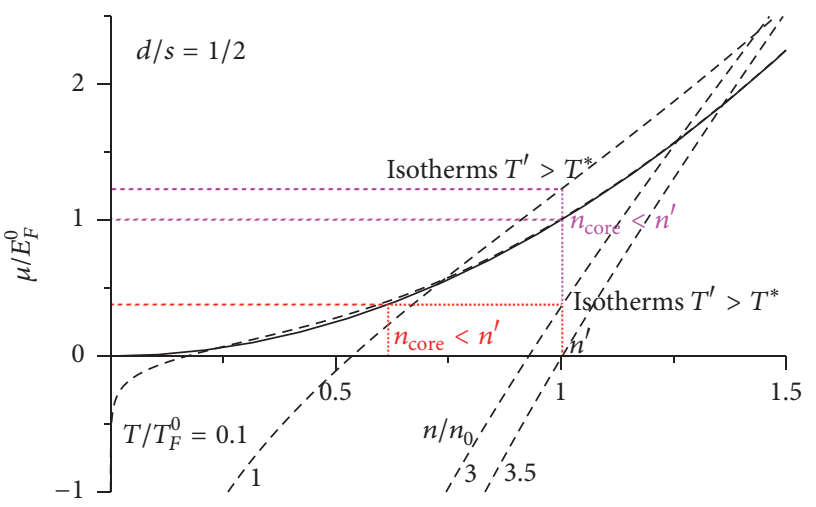

FIGURE 5: Isotherms in the plane $\mu$-n for the case $d / s=1 / 2$. Chemical potential and particle density are scaled with $E_{F}^{0}$ and $n_{0}$, respectively, which correspond to the Fermi energy of an IFG with an arbitrary particle density $n_{0}$. The thick line corresponds to the zero temperature isotherm, while thin-dashed lines label the isotherms with scaled temperatures $T / T_{F}^{0}=0.1,1,3$, and 3.5. For isotherms lying in the region $\mu>0$ but below the zero temperature isotherm it is possible to find $n_{\text {core }}$.

as follows [40]: for a given value of the system density, let us say $n^{\prime}$ and a temperature $T^{\prime} n_{\text {core }}$ are found on the $\mu-n$ plane as the value of $n$ that corresponds to the intersection of the horizontal line $\mu\left(T^{\prime}, n^{\prime}\right)$ with the isotherm $\mu_{0}(n)=$ $\mu(T=0, n)$ (thick line in Figure 5 corresponds to $d / s=1 / 2$ ). Necessarily, such a process cannot be performed at constant density implying an exchange of particles with and external reservoir in thermodynamic equilibrium with the system.

It is evident that no such intersection exists if $\mu\left(T^{\prime}, n^{\prime}\right)<$ 0 ; that is, no interpretation of a core can be formulated in the nondegenerate regime; however, a solution $n_{\text {core }} \leq n$ always

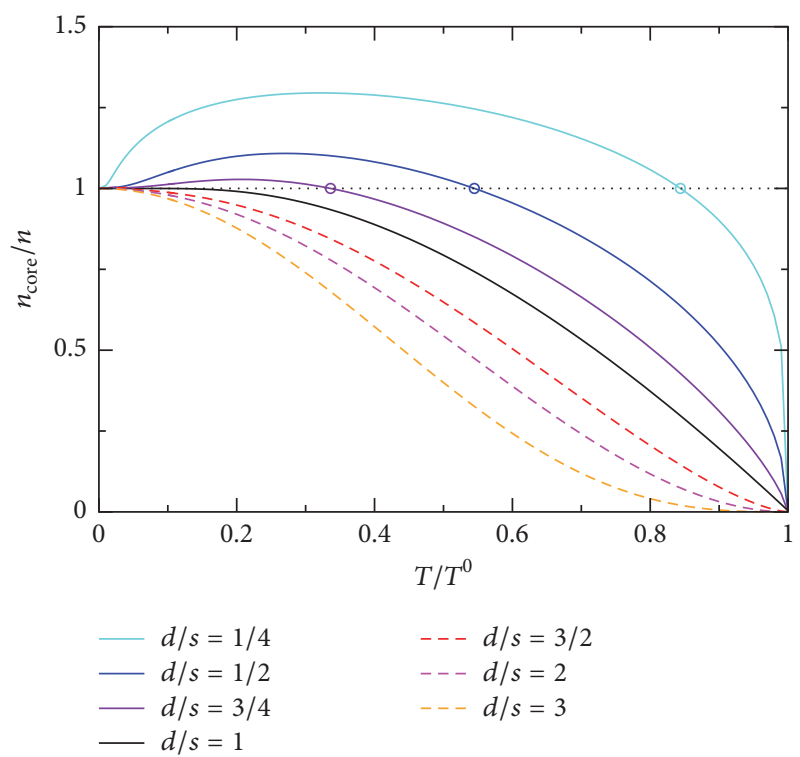

FIGURE 6: Fraction of particles in the Fermi-sphere-like condensate as function of temperature scaled with $T^{0}$ for different values of $d / s$.

exists for $\mu\left(T^{\prime}, n^{\prime}\right)>0$ and $d / s \geq 1$, since the isotherm $\mu_{0}(n)$ is a concave function of the particle density; in other words, isotherms $\mu(T, n)$ of higher temperature are situated below $\mu_{0}(n)$ (see [40] where the case $d / s=3 / 2$ is discussed). In contrast, for $d / s<1$, the zero temperature isotherm is a convex function of $n$ as shown in Figure 5, and two possibilities may happen: (i) if $T^{\prime}<T^{*}$ the intersection occurs at $n_{\text {core }}<n^{\prime}$ (dark-broken lines in Figure 5 for the case $d / s=1 / 2$ ); (ii) on the contrary, if $T^{\prime}>T^{*}$ the intersection occurs at $n_{\text {core }}>n^{\prime}$ as shown explicitly in the same figure. The dependence of the fraction $n_{\text {core }} / n$ on temperature is shown in Figure 6 for different values of $d / s$ and is explicitly given by the expression

$$
\frac{n_{\text {core }}}{n}=\left[\frac{\mu(T)}{E_{F}}\right]^{d / s} \quad \text { for } T \leq T^{0} .
$$

Notice that the nonmonotonic dependence of $\mu(T)$ for $d / s<1$ makes $n_{\text {core }} / n$ to reach the value 1 at the temperature $T^{*}$ (see circles in Figure 6).

\section{The Argument Energy-Entropy and the Meaning of $T^{*}$}

We now attempt to give a physical meaning to $\mu$ in the region where is larger than $E_{F}$, that is, in the interval of temperatures $\left[0, T^{*}\right]$. For this purpose we compute $\mu(T, \mathscr{V}, N)$ from the thermodynamic relation

$$
\mu(T, \mathscr{V}, N)=\left(\frac{\partial F}{\partial N}\right)_{T, \mathscr{V}},
$$

where $F=F(T, \mathscr{V}, N)$ stands for the Helmholtz free energy given by $F=-k_{B} T \ln Z_{N, \mathscr{V}}(\beta)=U-T S$ with $Z_{N, \mathscr{V}}(\beta)=$ $\sum_{E_{N, \mathscr{V}}} \exp \left\{-\beta E_{N, \mathscr{V}}\right\}$ the canonical partition function. The sum is made over the energies $E_{N, \mathscr{V}}$ of all possible configurations with exactly $N$ fermions in the volume $\mathscr{V}$. An advantage 
of expression (15) over the use of the relation (10) is that, at constant temperature and volume, the chemical potential measures the balance between the change of the internal energy and the heat exchanged when the number of particles in the system is varied from $N$ to $N+1$, making it suitable for the use of an energy-entropy argument [74]. Thus expression (15) provides a suitable operational definition, in the discrete case, of the chemical potential when only one particle is added isothermally to the system; namely $[47,72,75]$,

$$
\begin{aligned}
\mu(T, \mathscr{V}, N) & =\Delta F \equiv F(N+1, T, \mathscr{V})-F(N, T, \mathscr{V}), \\
& =k_{B} T \ln \left[\frac{Z_{N, \mathscr{V}}(\beta)}{Z_{N+1, \mathscr{V}}(\beta)}\right] .
\end{aligned}
$$

The rhs of expression (16a) can be explicitly written as $\Delta U-$ $T \Delta S$, where $\Delta U=U(T, \mathscr{V}, N+1)-U(T, \mathscr{V}, N)$ and $\Delta S=$ $S(T, \mathscr{V}, N+1)-S(T, \mathscr{V}, N)$ are the internal energy change of the system and the heat produced $T \Delta S$ when adding, isothermally, exactly one more fermion (expression (16a) arises from the forward difference discretization of the conventional definition (15). The physical interpretation is simple and the same for the backward difference: they simply give the change in the free energy when adding an extra particle to the system. Experimental realization of such situation is, for instance, Bose-Einstein condensation at constant temperature by increasing the particle number of the system [76] and injection of electrons into low-dimensional systems. The negative of such difference gives the change in the free energy when removing a particle from the system. Another definition of $\mu$ is given by the average of the forward and backward differences which is suitable when both processes, adding and removing a particle, are present at finite temperatures as occurs in the grand canonical ensemble).

At zero temperature, the chemical potential is given by the change in internal energy only, whose value coincides with the Fermi energy of $N+1$ fermions; that is,

$$
\mu(\mathscr{V}, N)=E_{F, N+1},
$$

where $E_{F, N}$ denotes the explicit dependence of the Fermi energy on the particle number. If this value is subtracted from (16a) we have that

$$
\Delta \mu=\Delta U^{\prime}-T \Delta S
$$

where $\Delta \mu=\mu(T, \mathscr{V}, N)-E_{F, N+1}$ and $\Delta U^{\prime}=\Delta U-E_{F, N+1}$. In this way, if for a given temperature we have that $\Delta \mu \leq 0$ (i.e., the chemical potential lies below the Fermi energy), then the relative change in the internal energy is smaller than the respective heat exchange by adding the particle. In other words, the effects of the addition of a particle to the system, in an isothermal way, are such that the entropic effects dominate over the energetic ones at that $T$. This argument accounts for the monotonic decreasing behavior of $\mu$ with $T$ and is equivalent with argument given in [41]. Further, if $\Delta \mu>0$ for a given $T$, then the energetic changes are the ones that dominate over the entropic ones, which give origin to the rise of $\mu$ above the Fermi energy as has been shown in the previous section. The temperature that separates both regimes
TABLE 2: The first 4 expressions for the canonical partition function are shown.

\begin{tabular}{lc}
\hline$N$ & $Z_{N}(\beta)$ \\
\hline 1 & $Z_{1}(\beta)$ \\
2 & $\frac{1}{2} Z_{1}^{2}(\beta)-\frac{1}{2} Z_{1}(2 \beta)$ \\
3 & $\frac{1}{6} Z_{1}^{3}(\beta)-\frac{1}{2} Z_{1}(\beta) Z_{1}(2 \beta)+\frac{1}{3} Z_{1}(3 \beta)$ \\
4 & $\frac{1}{24} Z_{1}^{4}(\beta)-\frac{1}{4} Z_{1}^{2}(\beta) Z_{1}(2 \beta)+\frac{1}{3} Z_{1}(\beta) Z_{1}(3 \beta)+$ \\
& $\frac{1}{8} Z_{1}^{2}(2 \beta)-\frac{1}{4} Z_{1}(4 \beta)$ \\
\hline
\end{tabular}

coincides with $T^{*}$, which is different from zero when $d / s<$ 1. This suggests the possibility of interpreting $T^{*}$ as a critical temperature at which a phase transition occurs.

In order to show the validity of these ideas we first use expression (16b) to compute $\mu$ in two distinct onedimensional systems each consisting of $N$ spinless fermions. One corresponds to the IFG trapped by a box-like potential $(d / s=1 / 2)$ and the other to the experimentally feasible system of and IFG trapped by a harmonic trap $(d / s=1)$. We show that, for the former case, $\mu$ rises above $E_{F, N+1}$ and eventually returns to its decreasing behavior as the system temperature is increased from zero. For the latter, we show that $\mu<E_{F, N+1}$ for all $T>0$.

For exactly $N$ noninteracting fermions, the partition function satisfies the recursive relation $[77,78]$.

$$
Z_{N}(\beta)=\frac{1}{N} \sum_{n=1}^{N}(-1)^{n+1} Z_{1}(n \beta) Z_{N-n}(\beta),
$$

where $Z_{1}(\beta)=\sum_{\varepsilon} \exp \{-\beta \varepsilon\}$ is the single-particle partition function with $\varepsilon$ being the single-particle energy spectrum and $Z_{0}(\beta) \equiv 1$.

Expression (19) can be reduced to the calculation of $Z_{1}(m \beta)$, with $m$ being a positive integer, by noting that $Z_{N}(\beta)$ can be written as a sum of the product over the distinct parts of all the partitions $\left\{\left(\lambda_{1}, \lambda_{2}, \ldots, \lambda_{r}\right)\right\}$ of $N$ (a partition is defined as a nonincreasing sequence of positive integers $\lambda_{1}, \lambda_{2}, \ldots, \lambda_{r}$ such that $\sum_{i=1}^{r} \eta_{i} \lambda_{i}=N$, where $\eta_{i}$ denotes the multiplicity of the part $\lambda_{i}$ in a given partition, that is, the partition $2+2+2+1$ of 7 ; the part 2 has multiplicity 3 ; see $[79$, pp.1]); thus

$$
\begin{aligned}
& Z_{N}(\beta) \\
& \quad=(-1)^{N} \sum_{\left\{\left(\lambda_{1}, \lambda_{2}, \ldots, \lambda_{r}\right)\right\}} \prod_{m=1}^{r} \frac{(-1)^{\eta_{m}}}{\lambda_{m}^{\eta_{m}} \eta_{m} !}\left[Z_{1}\left(\lambda_{m} \beta\right)\right]^{\eta_{m}} .
\end{aligned}
$$

The first four terms can be checked straightforwardly and are shown in Table 2.

Computationally, evaluation of expression (20) is faster than evaluating expression (19) since recursion is avoided and only the algorithm for computing the unrestricted partitions of the integer $N$ is needed. Such algorithm forms part of the MATHEMATICA software package distribution. The computation time and memory requirements grow with number of partitions $p(N)$ of $N$, which grows asymptotically as 
$\exp \{\sqrt{n}\}$ thus limiting computation to $N \sim 10$. Surprisingly, the calculation exhibits a fast convergence to the well-known result obtained from (3) for 64 particles (see Figure 7 for the box-like trap).

For the box potential in one dimension, the singleparticle partition function is given in terms of the Jacobi theta function $\vartheta_{3}(u, q)=1+2 \sum_{n=1}^{\infty} q^{n^{2}} \cos (2 n * u)$ as $Z_{1}(\beta)=$ $(1 / 2)\left[\vartheta_{3}\left(0, e^{-\beta \varepsilon_{0}}\right)-1\right]$, where the energy scale $\varepsilon_{0}$ in the argument of the exponential is $\hbar^{2} \pi^{2} / 2 m L^{2}$. For few particles, the chemical potential does not rise as $\left(T / T_{F}\right)^{2}$ as is expected from the grand canonical ensemble result, but it grows much more slower as is shown in the inset of Figure 7. This is consequence of the low temperature behavior of the partition function, which satisfies that $Z_{N} / Z_{N+1} \sim$ $e^{\beta E_{F, N+1}}\left[1+e^{-\beta(2 N+1) \varepsilon_{0}}\right]$ for $T / T_{F} \ll 1$, leading thus to $\Delta \mu \sim$ $k_{B} T e^{-\beta(2 N+1) \varepsilon_{0}}$.

For the harmonic potential in dimension one, an exact analytical expression for $Z_{N}(\beta)$ is known [24, 80]; namely,

$$
Z_{N}(\beta)=\exp \left[-N^{2} \beta \frac{\hbar \omega}{2}\right] \prod_{j=1}^{N}[1-\exp (-\beta \hbar \omega j)]^{-1} .
$$

A direct application of (16b) leads to

$$
\begin{aligned}
\mu= & E_{F, N+1} \\
& +k_{B} T \ln \left[1-\exp \left(-\beta E_{F, N+1}\right) \exp \left(-\frac{\beta \hbar \omega}{2}\right)\right] .
\end{aligned}
$$

Clearly (22) is a monotonically decreasing function of $T$ agreeing with the grand canonical ensemble result; expression $\mu=E_{F}+k_{B} T \ln \left[1-e^{-T_{F} / T}\right]$ is recovered in the limit $N \rightarrow \infty$.

\section{Conclusions and Final Remarks}

In this paper we have presented a discussion on the meaning of the nonmonotonic dependence on temperature of the thermodynamics properties of low-dimensional, trapped, IFGs, with focus on the chemical potential (a similar behavior has been predicted for weakly repulsively interacting Bose gases [81] in that a hard core Bose gas behaves, at least qualitatively, as an ideal Fermi gas). The parameter used to characterize the trapping and dimensionality $d$ of the system is merely $d / s$ that explicitly appears in the single-particle density of states (1). Thus low-dimensional trapped systems are characterized by values of $d / s<1$. In this range of values, the chemical potential, the specific heat at constant volume, and the isothermal compressibility exhibit a nonmonotonic dependence on temperature which have been characterized by the temperatures $T_{\mu}, T_{C_{\mathscr{Y}}}$, and $T_{\kappa_{T}}[60]$, respectively. We also have computed $T^{0}$ as function of $d / s[28,40]$ and introduced a new characteristic temperature $T^{*} \leq T^{0}$, which corresponds to the nonzero value of the temperature at which $\mu\left(T^{*}, \mathscr{V}, N\right)=E_{F}$.

We found that $T^{*}$ marks the temperature at which the particle density of a Fermi-like core $n_{\text {core }}$ that starts forming $T^{0}$ saturates at the value of the total particle density of the

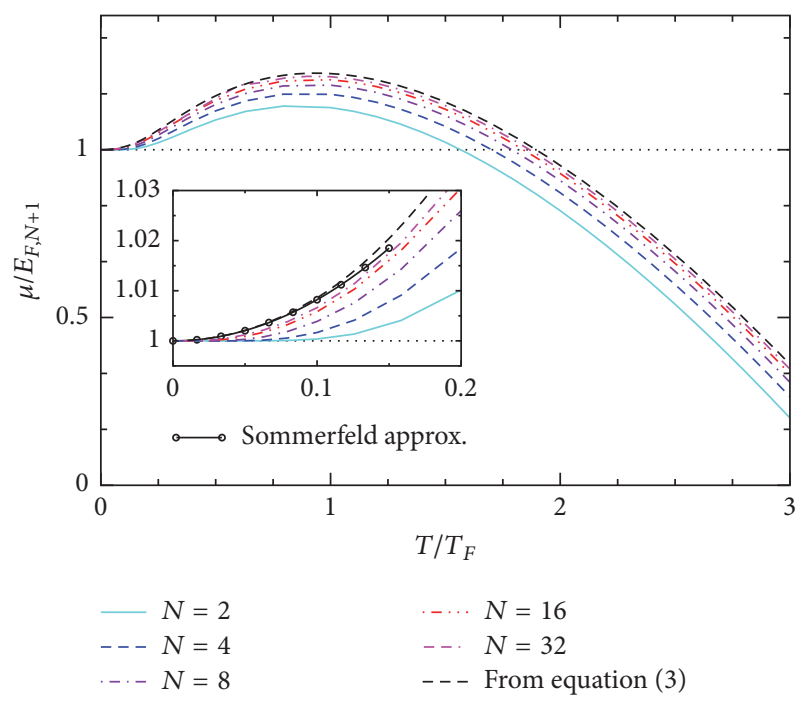

FIgURE 7: The chemical potential (16b), scaled with $E_{F, N+1}$ as function of the dimensionless temperature $T / T_{F}$, with $T_{F}=$ $E_{F, N+1} / k_{B}$, for the number of particles $N=2,4,8,16$, and 32 . Note that the results with 32 particles (double dashed-dotted line) are close to the grand canonical ensemble result (black line in long dashes) computed from (3). A comparison with the Sommerfeld approximation for low temperatures is shown in the inset.

system $n$. This suggests that $T^{*}$ can be considered as the relevant temperature of the isotropically trapped IFG, as is supported by the energy-entropy-like argument presented in Section 5. The region in the $\mu-T$ plane, for which $\mu>E_{F}$ for $T \leq T^{*}$, represents the set of thermodynamic states for which the change in the Helmholtz free energy, when increasing the particle density of the system, is dominated by the changes of the internal energy and would correspond to an ordered phase. In the complementary region for which $T \geq T^{*}$, the thermodynamic states are characterized by changes in $F(T, \mathscr{V}, N)$ dominated by heat exchange by changing entropy and can be considered as a "disordered phase." Though, heuristic energy-entropy arguments have been used to uncover the possibility of a phase transition [74], we want to emphasize that we are not claiming the existence of a phase transition in the IFG, on the basis that thermodynamic quantities do not show a singular behavior of the thermodynamic susceptibilities at $T^{*}$.

Though the chemical potential is not directly measured in current experiments, development on imaging techniques of ultracold gases [82-85] has opened the possibility of experimentalists to measure the local particle density in situ and from the data to extract $\mu$ and $T$.

\section{Competing Interests}

The author declares that they have no competing interests.

\section{Acknowledgments}

The author wants to express their gratitude to Mauricio Fortes and Miguel Angel Solís for valuable discussions and to Omar 
Piña Pérez for helping in generating Figure 2. Support by UNAM-DGAPA PAPIIT-IN113114 is acknowledged.

\section{References}

[1] W. Pauli, "Über den zusammenhang des abschlusses der elektronengruppen im atom mit der komplexstruktur der spektren," Zeitschrift für Physik, vol. 31, no. 1, pp. 765-783, 1925.

[2] E. Fermi, "Sulla quantizzazione del gas perfetto monoatomico," Rendiconti Lincei, vol. 3, pp. 145-149, 1926.

[3] P. Dirac, "On the theory of quantum mechanics," Proceedings of the Royal Society of London A, vol. 112, no. 762, p. 661, 1926.

[4] J. Sólyom, "The Fermi gas model of one-dimensional conductors," Advances in Physics, vol. 28, no. 2, pp. 201-303, 1979.

[5] J. Voit, "One-dimensional Fermi liquids," Reports on Progress in Physics, vol. 58, no. 9, 1995.

[6] X.-W. Guan, M. T. Batchelor, and C. Lee, "Fermi gases in one dimension: from bethe ansatz to experiments," Reviews of Modern Physics, vol. 85, no. 4, pp. 1633-1691, 2013.

[7] B. DeMarco and D. S. Jin, "Onset of Fermi degeneracy in a trapped atomic gas," Science, vol. 285, no. 5434, pp. 1703-1706, 1999.

[8] A. G. Truscott, K. E. Strecker, W. I. McAlexander, G. B. Partridge, and R. G. Hulet, "Observation of fermi pressure in a gas of trapped atoms," Science, vol. 291, no. 5513, pp. 2570-2572, 2001.

[9] S. R. Granade, M. E. Gehm, K. M. O’Hara, and J. E. Thomas, "All-optical production of a degenerate Fermi gas", Physical Review Letters, vol. 88, no. 12, Article ID 1204051, 2002.

[10] Z. Hadzibabic, S. Gupta, C. A. Stan et al., "Fiftyfold improvement in the number of quantum degenerate fermionic atoms," Physical Review Letters, vol. 91, no. 16, Article ID 160401, 2003.

[11] T. Fukuhara, Y. Takasu, M. Kumakura, and Y. Takahashi, "Degenerate fermi gases of ytterbium," Physical Review Letters, vol. 98, no. 3, Article ID 030401, 2007.

[12] C. Chin, M. Bartenstein, A. Altmeyer et al., "Observation of the pairing gap in a strongly interacting Fermi gas," Science, vol. 305, no. 5687, pp. 1128-1130, 2004.

[13] G. K. Campbell, M. M. Boyd, J. W. Thomsen et al., "Probing interactions between ultracold fermions," Science, vol. 324, no. 5925, pp. 360-363, 2009.

[14] M. W. Zwierlein, C. H. Schunck, A. Schirotzek, and W. Ketterle, "Direct observation of the superfluid phase transition in ultracold Fermi gases," Nature, vol. 442, no. 7098, pp. 54-58, 2006.

[15] Y.-I. Shin, A. Schirotzek, C. H. Schunck, and W. Ketterle, "Realization of a strongly interacting bose-fermi mixture from a twocomponent fermi gas," Physical Review Letters, vol. 101, no. 7, Article ID 070404, 2008.

[16] D. A. Butts and D. S. Rokhsar, “Trapped Fermi gases," Physical Review A, vol. 55, no. 6, pp. 4346-4350, 1997.

[17] J. Schneider and H. Wallis, "Mesoscopic Fermi gas in a harmonic trap," Physical Review A-Atomic, Molecular, and Optical Physics, vol. 57, no. 2, pp. 1253-1259, 1998.

[18] M. Li, Z. Yan, J. Chen, L. Chen, and C. Chen, “Thermodynamic properties of an ideal Fermi gas in an external potential with $U$ = brt in any dimensional space," Physical Review A, vol. 58, no. 2, pp. 1445-1449, 1998.

[19] A. Thislagam, "Dimensionality dependence of Pauli blocking effects in semiconductor quantum wells," Journal of Physics and Chemistry of Solids, vol. 60, pp. 497-502, 1999.
[20] P. Vignolo, A. Minguzzi, and M. P. Tosi, "Exact particle and kinetic-energy densities for one-dimensional confined gases of noninteracting fermions," Physical Review Letters, vol. 85, no. 14, article 2850, 2000.

[21] M. Brack and B. P. van Zyl, "Simple analytical particle and kinetic energy densities for a dilute fermionic gas in a $d$ dimensional harmonic trap," Physical Review Letters, vol. 86, no. 8, pp. 1574-1577, 2001.

[22] G. M. Bruun and C. W. Clark, "Ideal gases in time-dependent traps," Physical Review A, vol. 61, no. 6, Article ID 061601, 2000.

[23] F. Gleisberg, W. Wonneberger, U. Schlöder, and C. Zimmermann, "Noninteracting fermions in a one-dimensional harmonic atom trap: exact one-particle properties at zero temperature," Physical Review A, vol. 62, no. 6, Article ID 063602, 2000.

[24] M. N. Tran, M. V. Murthy, and R. K. Bhaduri, "Ground-state fluctuations in finite Fermi systems," Physical Review E, vol. 63, no. 3, Article ID 031105, 2001.

[25] Z. Akdeniz, P. Vignolo, A. Minguzzi, and M. P. Tosi, “Temperature dependence of density profiles for a cloud of noninteracting fermions moving inside a harmonic trap in one dimension," Physical Review A, vol. 66, no. 5, Article ID 055601, 2002.

[26] P. Vignolo and A. Minguzzi, "Shell structure in the density profiles for noninteracting fermions in anisotropic harmonic confinement," Physical Review A, vol. 67, no. 5, Article ID 053601, 2003.

[27] M. Grether, M. Fortes, M. De Llano et al., "Harmonically trapped ideal quantum gases," European Physical Journal D, vol. 23, no. 1, pp. 117-124, 2003.

[28] D.-V. Anghel, “Condensation in ideal Fermi gases," Journal of Physics A: Mathematical and General, vol. 36, no. 46, pp. L577L583, 2003.

[29] M. N. Tran, "Exact ground-state number fluctuations of trapped ideal and interacting fermions," Journal of Physics. A. Mathematical and General, vol. 36, no. 4, pp. 961-973, 2003.

[30] B. P. Van Zyl, R. K. Bhaduri, A. Suzuki, and M. Brack, "Some exact results for a trapped quantum gas at finite temperature," Physical Review A, vol. 67, no. 2, Article ID 023609, 2003.

[31] E. J. Mueller, "Density profile of a harmonically trapped ideal Fermi gas in arbitrary dimension," Physical Review Letters, vol. 93, no. 19, Article ID 190404, 2004.

[32] D.-V. Anghel, O. Fefelov, and Y. M. Galperin, "Fluctuations of the Fermi condensate in ideal gases," Journal of Physics. A. Mathematical and General, vol. 38, no. 43, article 9405, 2005.

[33] D.-Y. Song, "Exact coherent states of a noninteracting Fermi gas in a harmonic trap," Physical Review A, vol. 74, no. 5, Article ID $051602,2006$.

[34] M. M. Faruk and G. M. Bhuiyan, "Thermodynamics of ideal Fermi gas under generic power law potential in d-dimensions," Acta Physica Polonica B, vol. 46, no. 12, pp. 2419-2569, 2015.

[35] B. DeMarco, S. B. Papp, and D. S. Jin, "Pauli blocking of collisions in a quantum degenerate atomic Fermi gas," Physical Review Letters, vol. 86, no. 24, pp. 5409-5412, 2001.

[36] D. Stamper-Kurn, "Shifting entropy elsewhere," Physics, vol. 2, article no. 80, 2009.

[37] J. Catani, G. Barontini, G. Lamporesi et al., "Entropy exchange in a mixture of ultracold atoms," Physical Review Letters, vol. 103, no. 14, Article ID 140401, 2009.

[38] J.-S. Bernier, C. Kollath, A. Georges et al., "Cooling fermionic atoms in optical lattices by shaping the confinement," Physical Review A, vol. 79, no. 6, Article ID 061601, 2009. 
[39] T. Paiva, R. Scalettar, M. Randeria, and N. Trivedi, "Fermions in 2D optical lattices: temperature and entropy scales for observing antiferromagnetism and superfluidity," Physical Review Letters, vol. 104, no. 6, Article ID 066406, 2010.

[40] V. Romero-Rochin, "Phase diagram of quantum fluids. The role of the chemical potential and the phenomenon of condensation," https://arxiv.org/abs/1109.1815.

[41] G. Cook and R. H. Dickerson, "Understanding the chemical potential," American Journal of Physics, vol. 63, no. 8, pp. 737742, 1995.

[42] R. Baierlein, "The elusive chemical potential," American Journal of Physics, vol. 69, no. 4, pp. 423-434, 2001.

[43] G. Job and F. Herrmann, "Chemical potential-a quantity in search of recognition," European Journal of Physics, vol. 27, no. 2, pp. 353-371, 2006.

[44] C. E. Mungan, "Chemical potential of one-dimensional simple harmonic oscillators," European Journal of Physics, vol. 30, no. 5, pp. 1131-1136, 2009.

[45] M. R. A. Shegelski, "New result for the chemical potential of intrinsic semiconductors: low-temperature breakdown of the Fermi-Dirac distribution function," Solid State Communications, vol. 58, no. 6, pp. 351-354, 1986.

[46] P. T. Landsberg and D. C. Browne, "The chemical potential of an intrinsic semiconductor near T $=0$," Solid State Communications, vol. 62, no. 3, pp. 207-208, 1987.

[47] M. R. A. Shegelski, "The chemical potential of an ideal intrinsic semiconductor," American Journal of Physics, vol. 72, no. 5, pp. 676-678, 2004.

[48] T. A. Kaplan, "The chemical potential," Journal of Statistical Physics, vol. 122, no. 6, pp. 1237-1260, 2006.

[49] S. Panda and B. K. Panda, "Chemical potential and internal energy of the noninteracting Fermi gas in fractional-dimensional space," Pramana-Journal of Physics, vol. 75, no. 3, pp. 393-402, 2010.

[50] F. J. Sevilla and L. Olivares-Quiroz, "Chemical potential for the interacting classical gas and the ideal quantum gas obeying a generalized exclusion principle," European Journal of Physics, vol. 33, no. 3, article 709, 2012.

[51] F. J. Sevilla and O. Piña, "Thermodynamics of the relativistic fermi gas in D dimensions," https://arxiv.org/abs/1407.7187.

[52] P. Salas and M. A. Solís, "Trapping effect of periodic structures on the thermodynamic properties of a fermi gas," Journal of Low Temperature Physics, vol. 175, no. 1-2, pp. 427-434, 2014.

[53] P. C. Hohenberg, "Existence of long-range order in one and two dimensions," Physical Review, vol. 158, no. 2, pp. 383-386, 1967.

[54] M. H. Lee, "Equivalence of ideal gases in two dimensions and Landen's relations," Physical Review E, vol. 55, no. 2, article 1518, 1997.

[55] R. M. May, "Quantum statistics of ideal gases in two dimensions," Physical Review A, vol. 135, pp. A1515-A1518, 1964.

[56] D.-V. Anghel, "Gases in two dimensions: universal thermodynamics and its consequences," Journal of Physics A: Mathematical and General, vol. 35, no. 34, pp. 7255-7267, 2002.

[57] K. Schönhammer and V. Meden, "Fermion-Boson transmutation and comparison of statistical ensembles in one dimension," American Journal of Physics, vol. 64, no. 9, pp. 1168-1176, 1996.

[58] M. Crescimanno and A. S. Landsberg, "Spectral equivalence of bosons and fermions in one-dimensional harmonic potentials," Physical Review A, vol. 63, no. 3, Article ID 035601, 2001.
[59] R. K. Pathria, "Similarities and differences between Bose and Fermi gases," Physical Review E, vol. 57, no. 3, pp. 2697-2702, 1998.

[60] M. Grether, M. de Llano, and M. A. Solís, "Anomalous behavior of ideal Fermi gas below two dimensions," European Physical Journal D, vol. 25, no. 3, pp. 287-291, 2003.

[61] E. Cetina, F. Magana, and A. A. Valladares, "The free-electron gas in n dimensions," American Journal of Physics, vol. 45, no. 10, pp. 960-963, 1977.

[62] M. H. Lee, "Polylogarithmic analysis of chemical potential and fluctuations in a $D$-dimensional free Fermi gas at low temperatures," Journal of Mathematical Physics, vol. 36, no. 3, pp. 1217-1229, 1995.

[63] M. Grether, M. De Llano, and M. H. Lee, "Anomalous behavior of ideal fermi gas below 2D: the "ideal quantum dot" and the paul exclusion principle," International Journal of Modern Physics B, vol. 23, no. 20-21, pp. 4121-4128, 2009.

[64] I. Chávez, M. Grether, and M. De Llano, "Low-dimensional Fermi and Bose gases," Physica E, vol. 44, no. 2, pp. 394-399, 2011.

[65] V. C. Aguilera-Navarro, M. De Llano, and M. A. Solís, "BoseEinstein condensation for general dispersion relations," European Journal of Physics, vol. 20, no. 3, pp. 177-182, 1999.

[66] V. Bagnato, D. E. Pritchard, and D. Kleppner, "Bose-Einstein condensation in an external potential," Physical Review A, vol. 35 , no. 10 , pp. $4354-4358,1987$.

[67] R. K. Pathria, Statistical Mechanics, Elsevier, Oxford, UK, 2nd edition, 1996.

[68] K. Huang, Statistical Mechanics, John Wiley \& Sons, 2nd edition, 1987.

[69] N. Sandoval-Figueroa and V. Romero-Rochín, "Thermodynamics of trapped gases: generalized mechanical variables, equation of state, and heat capacity," Physical Review E, vol. 78, no. 6, Article ID 061129, 2008.

[70] S. R. de Groot, G. J. Hooyman, and C. A. Ten Seldam, "In difference with a gas in a $3 \mathrm{D}$-box potential where ratio N/V is well defined as $\mathrm{V}$ being the box volume is well defined too, in the gas trapped by harmonic potentials the thermodynamic limit is defined through the limit of $\omega \rightarrow 0$ and $N \rightarrow \infty$ such that the product $\omega 3 \mathrm{~N}$ is constant as has been discussed in the literature see for instance," Proceedings of the Royal Society of London. Series A, vol. 203, article 266, 1950.

[71] L. Lewin, Dilogarithms and Associated Functions, McDonald, London, UK, 1958.

[72] N. W. Ashcroft and N. D. Mermin, Solid State Physics, Saunders, Philadelphia, Pa, USA, 1976.

[73] T. P. Meyrath, F. Schreck, J. L. Hanssen, C.-S. Chuu, and M. G. Raizen, "Bose-Einstein condensate in a box," Physical Review A, vol. 71, no. 4, Article ID 041604, 2005.

[74] B. Simon and A. D. Sokal, "Rigorous entropy-energy arguments," Journal of Statistical Physics, vol. 25, no. 4, pp. 679-694, 1981.

[75] J. Tobochnik, H. Gould, and J. Machta, "Understanding temperature and chemical potential using computer simulations," American Journal of Physics, vol. 73, no. 8, pp. 708-716, 2005.

[76] M. Erhard, H. Schmaljohann, J. Kronjäger, K. Bongs, and K. Sengstock, "Bose-Einstein condensation at constant temperature," Physical Review A, vol. 70, no. 3, Article ID 031602, 2004.

[77] P. Borrmann and G. Franke, "Recursion formulas for quantum statistical partition functions," The Journal of Chemical Physics, vol. 98, no. 3, pp. 2484-2485, 1993. 
[78] S. Pratt, "Canonical and microcanonical calculations for Fermi systems," Physical Review Letters, vol. 84, no. 19, pp. 4255-4259, 2000.

[79] G. E. Andrews, The Theory of Partitions, University of Cambridge, Cambridge, UK, 1998.

[80] M. N. Tran, M. V. Murthy, and R. K. Bhaduri, "On the quantum density of states and partitioning an integer," Annals of Physics, vol. 311, no. 1, pp. 204-219, 2004.

[81] Z. Zhang, H. Fu, J. Chen, and M. Li, "Chemical potential of a trapped interacting Bose gas," Physical Review A, vol. 79, no. 5, Article ID 055603, 2009.

[82] T.-L. Ho and Q. Zhou, "Obtaining the phase diagram and thermodynamic quantities of bulk systems from the densities of trapped gases," Nature Physics, vol. 6, no. 2, pp. 131-134, 2010.

[83] T. Müller, B. Zimmermann, J. Meineke, J.-P. Brantut, T. Esslinger, and H. Moritz, "Local observation of antibunching in a trapped fermi gas," Physical Review Letters, vol. 105, no. 4, Article ID 040401, 2010.

[84] S. Nascimbène, N. Navon, K. J. Jiang, F. Chevy, and C. Salomon, "Exploring the thermodynamics of a universal Fermi gas," Nature, vol. 463, no. 7284, pp. 1057-1060, 2010.

[85] C. Sanner, E. J. Su, A. Keshet et al., "Suppression of density fluctuations in a quantum degenerate fermi gas," Physical Review Letters, vol. 105, no. 4, Article ID 040402, 2010. 

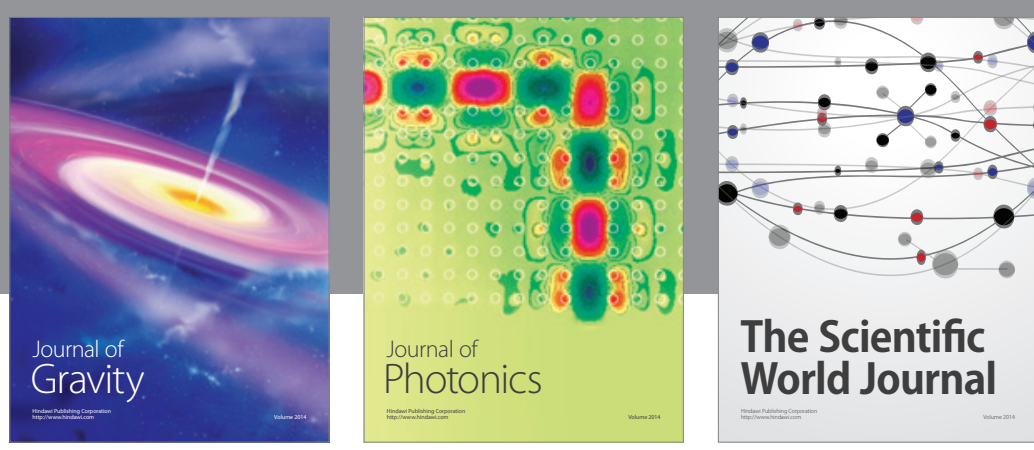

The Scientific World Journal
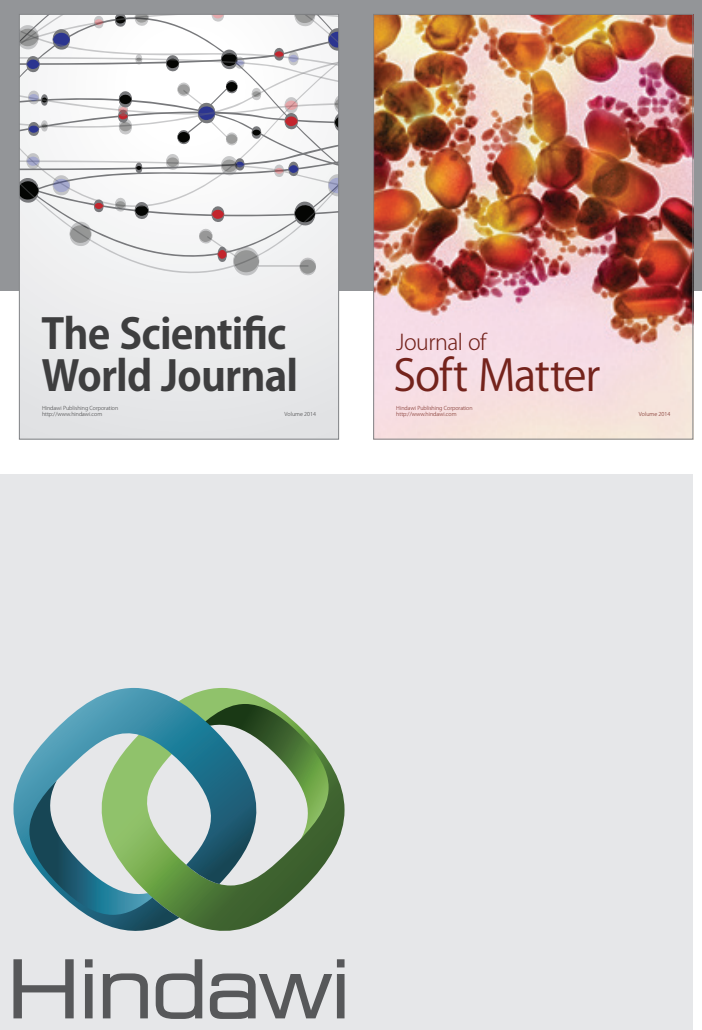

Submit your manuscripts at

https://www.hindawi.com
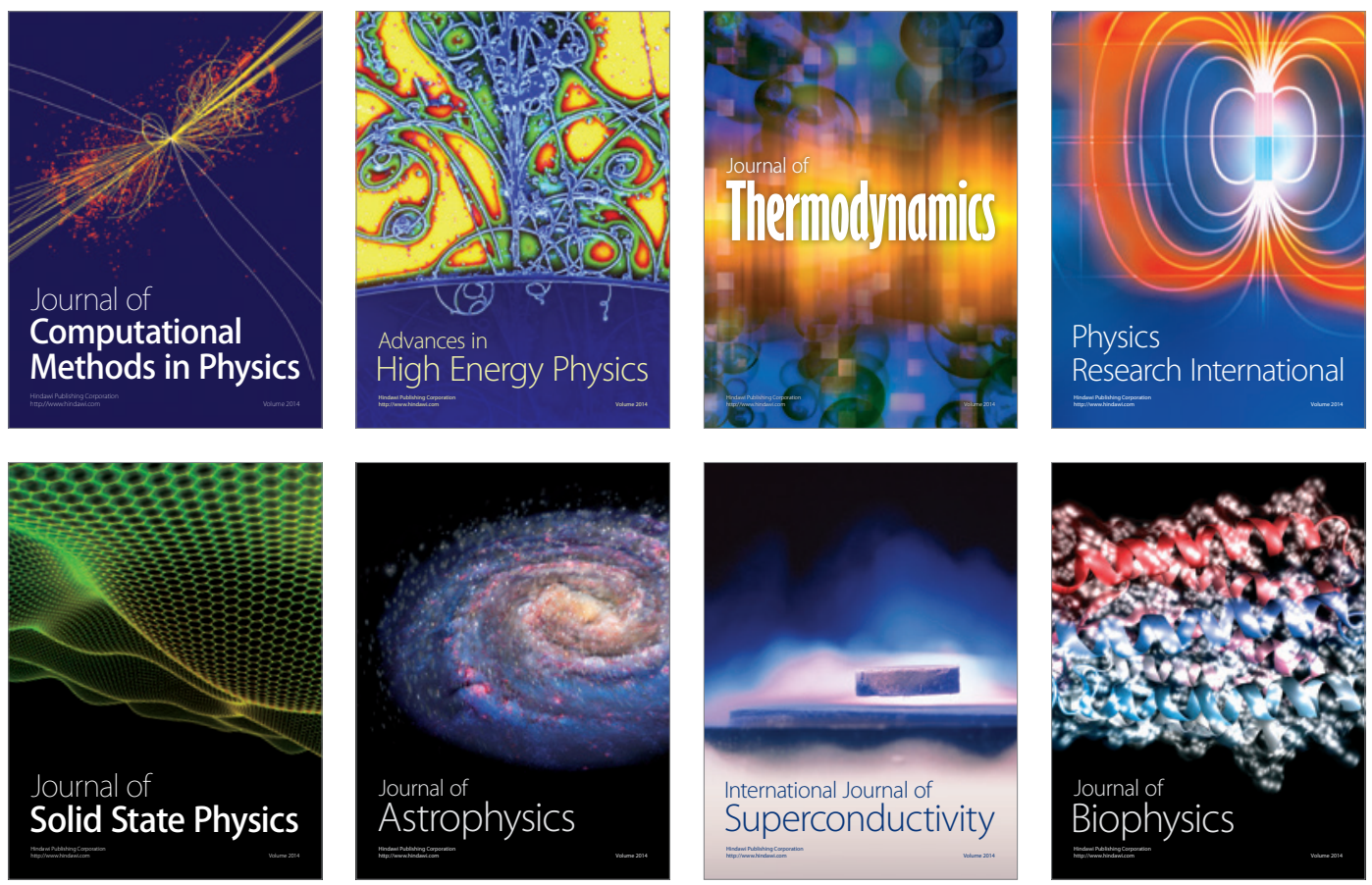
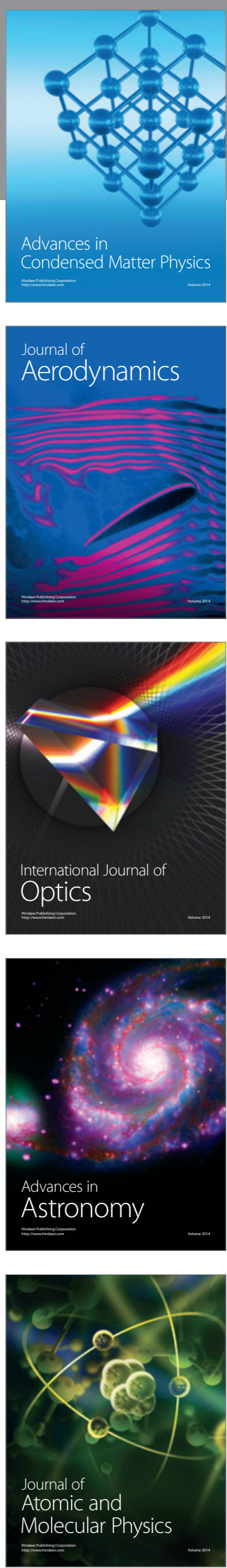